\title{
Blood Pressure Control in Patients with Chronic Kidney Disease
}

\author{
Kouichi Utsumi, Ken-ichiro Katsura, Yasuhiko Iino and Yasuo Katayama \\ Department of Neurological, Nephrological, and Rheumatological Science, Graduate School of Medicine, \\ Nippon Medical School
}

\begin{abstract}
Chronic kidney disease (CKD) is defined as either kidney damage or an estimated glomerular filtration rate (eGFR) of less than $60 \mathrm{~mL} / \mathrm{min} / 1.73 \mathrm{~m}^{2}$ for more than 3 months. Kidney damage is defined as pathological abnormalities or markers of damage, including abnormalities in blood or urine tests or imaging studies. CKD is classified as stage 1 to 5 on the basis of eGFR. Cardiovascular disease (CVD) carries a reciprocal risk of loss of kidney function in patients with chronic kidney disease (CKD) and with the development of kidney disease. CVD is a major cause of morbidity and mortality in patients with CKD. Blood pressure control in patients with CKD aims to prevent CVD and provide renoprotection. The reninangiotensin system (RAS) is involved in every stage of the progression of CKD and is, therefore, a critical link in the pathologic relationship between hypertension and renal disease. The first-line agents for controlling blood pressure are inhibitors of the RAS: angiotensinconverting enzyme inhibitors and angiotensin II receptor blockers. These agents have been shown to have renoprotective effects in addition to their ability to control bood pressure. In CKD, the target blood pressure is less than $130 / 80 \mathrm{~mm} \mathrm{Hg}$, or $125 / 75 \mathrm{~mm} \mathrm{Hg}$, if amount of urinary protein is more than $1 \mathrm{~g}$ /day. To achieve the target blood pressure, other classes of antihypertensive agents, such as diuretics and calcium channel blockers, should be administered in addition to angiotensin-converting enzyme inhibitors and angiotensin II receptor blockers.
\end{abstract}

(J Nippon Med Sch 2012; 79: 111-114)

Key words: chronic kidney disease, cardiovascular disease, renin-angiotensin system, angiotensin-converting enzyme inhibitors, angiotensin II receptor blockers

\section{Definition and Stages of Chronic Kidney Disease}

Chronic kidney disease (CKD) is defined as either kidney damage or an estimated glomerular filtration rate (eGFR) of less than $60 \mathrm{~mL} / \mathrm{min} / 1.73 \mathrm{~m}^{2}$ for more than 3 months (Table 1). Kidney damage is defined as pathologic abnormalities or markers of damage, including abnormalities in blood or urine tests or imaging studies; proteinuria is especially important. The classification of individuals is based on the presence or absence of markers of kidney disease and the eGFR (Table 2). Higher stages represent lower eGFR.

Correspondence to Kouichi Utsumi, MD, Department of Neurological, Nephrological, and Rheumatological Science, Graduate School of Medicine, Nippon Medical School, 1-1-5 Sendagi, Bunkyo-ku, Tokyo 113-8603, Japan

E-mail: utsumi@nms.ac.jp

Journal Website (http://www.nms.ac.jp/jnms/) 
Table 1 Definition of Chronic Kidney Disease

1. Kidney damage for $\geqq 3$ months, as defined by structural or functional abnormalities of the kidney, with or without decreased GFR, manifest by either: pathological abnormalities or marked of kidney damage, including abnormalities in the composition of the blood or urine, or abnormalities in imaging test

2. GFR $<60 \mathrm{~mL} / \mathrm{min} / 1.73 \mathrm{~m}^{2}$ for $\geqq 3$ months, with or without kidney damage

Ref (1)

\section{Progression of Renal Dysfunction}

There are many causes of renal dysfunction, including genetic abnormalities, autoimmune disease, toxic exposures, infections, and trauma, but hypertension and diabetes are among the most common $^{2}$. Because the primary function of the kidney is to filter the blood, thereby regulating blood volume and pressure and eliminating unwanted molecules while retaining essential ones, deterioration in renal function is reflected in the decreasing ability of the kidney to effectively filter the blood. The decline in function is seen as a progressive decrease in GFR. Hypertension is a major risk factor for progression of CKD and, as such, is an important therapeutic target.

Proteinuria results from glomerular injury that allows albumin and other large serum proteins to gain access to the mesangium and tubular fluid ${ }^{3}$. The magnitude of proteinuria generally correlates with the risk of progression to end-stage renal disease. In addition to being a facet of the natural history of CKD, proteinuria appears to reflect generalized system endothelial injury. In some animal models of CKD, proteinuria has been shown to be associated with the release of inflammatory mediators, which promote inflammation, culminating in the development of glomerular and tubulointerstitial fibrosis and kidney failure ${ }^{4}$.

\section{CKD and Cardiovascular Disease}

In Japan and Western countries, studies have shown that CKD is a risk factor for both the progression of end stage renal failure and the
Table 2 Stages of Chronic Kidney Disease

\begin{tabular}{lll}
\hline Stage & \multicolumn{1}{c}{ Description } & \multicolumn{1}{c}{$\begin{array}{c}\mathrm{GFR} \\
\left(\mathrm{mL} / \mathrm{min} / 1.73 \mathrm{~m}^{2}\right)\end{array}$} \\
\hline 1 & $\begin{array}{l}\text { Kidney damage with } \\
\text { or normal } \uparrow \text { GFR }\end{array}$ & $\geqq 90$ \\
2 & $\begin{array}{l}\text { Kidney damage with } \\
\text { mild } \downarrow \text { GFR }\end{array}$ & $60-90$ \\
3 & Moderate $\downarrow$ GFR & $30-59$ \\
4 & Severe $\downarrow$ GFR & $15-29$ \\
5 & Kidney failure & $<15$ (or dialysis) \\
\hline & & \multicolumn{2}{c}{ Ref (1) }
\end{tabular}

development of cardiovascular disease (CVD) $)^{5-7}$. Urinary protein, even at low levels, is a marker for CVD risk and for renal dysfunction ${ }^{6}$. In the general population without hypertension or diabetes, the presence of CKD, such as albuminuria and decrease in GFR in mild, has been shown to be a risk factor for $\mathrm{CVD}^{6}$. In a recent study, the rate of renal replacement therapy over the 5 -year observation period was $1.1 \%, 1.3 \%$, and $19.9 \%$, respectively, for the National Kidney Foundation Kidney Disease Outcomes Quality Initiative stages 2, 3, and 4, but that the mortality rate was $19.5 \%, 24.3 \%$, and $45.7 \%$. Thus, death was far more common than dialysis at all stages ${ }^{7}$. In particular, CVD was more prevalent in the patients who $\operatorname{died}^{7}$. Therefore, in patients with CKD, attention must always be paid to the development of CVD. In addition, it has been reported that renal function is decreased in patients with $\mathrm{CVD}^{8}$. Furthermore, renal anemia is an independent risk factor for CVD in patients with CKD. Treatment of the risk factors of CKD, such as hypertension and diabetes mellitus, aims to prevent progressive CKD and to CVD.

\section{Pharmacologic Blockade of the Renin-angiotensin System}

The renin-angiotensin system (RAS) is involved in every stage of the progression of CKD and is, therefore, a critical link in the pathologic relationship between hypertension and renal disease. Regulation of extracellular fluid volume, sodium and water homeostasis, and electrolyte balance are the primary functions of the RAS. The sympathetic nervous system and vasoactive hormones work with the RAS to coordinate its hemodynamic effects and 
thereby maintain blood pressure. Angiotensinconverting enzyme inhibitors (ACEIs) block the conversion of angiotensin I (A I) to angiotensin II (A II), the peptide thought to initiate most of the deleterious effects of the RAS. Because ACEIs also metabolize bradykinin, a direct vasodilator, and promote release of the vasodilators $\mathrm{NO}$ and prostacyclin, a second effect of ACEIs is the reduction of bradykinin degradation'. However, studies have shown that levels of circulating A II return to pretreatment levels in patients receiving long-term ACEI therapy. This process, known as ACE escape, is attributed to alternate pathways for A II, such as those involving cathepsin G and elastase, which may directly convert angiotensinogen to $\mathrm{A}$ II, and chymases and cathepsin $\mathrm{G}$, which may convert $\mathrm{A}$ I to $\mathrm{A} \mathrm{II}^{10,11}$. On the other hand, A II receptor blockers (ARBs) selectively block the binding of A II to A II type I receptors without interfering with the binding of $A$ II to A II type II (AT2) receptors, thereby, allowing potentially beneficial AT2-mediated effects (vasodilation, inhibition of cell growth, improved endotherial function, and inhibition of collagen deposition) ${ }^{9}$. Theoretically, the combination of an ACEI and an ARB provides more complete RAS inhibition while providing the beneficial effects of increased bradykinin levels and AT2 receptor activation.

\section{Rationale for Combination Therapy}

Their complementary mechanisms of action in inhibiting the RAS make dual blockade of the RAS system with ACEIs and ARBs an attractive possibility for further improving the renoprotective effects observed with monotherapy. In part, the rationale for evaluating submaximal doses of ACEIs and ARBs in combination therapy is the potential for high-dose ACE or ARB monotherapy to worsen renal function, hypotension, or hyperkalemia. A recent meta-analysis of 14 relevant trials evaluated the safety of the combination of an ACEI and an $\mathrm{ARB}^{12}$. In another recent meta-analysis of combination therapy in diabetic nephropathy, combination therapy with submaximal doses of
ACEIs and ARBs was found to reduce 24-hour proteinuria better than does an ACEI alone ${ }^{13}$. Benefit was achieved with only small effects on GFR, serum creatinine level, potassium, and blood pressure ${ }^{13}$. With a median follow-up period of 56 months, the Ongoing Telmisartan Alone and in Combination with Ramipril Global Endpoint Trial (ONTARGET) evaluated combination therapy with telmisartan and ramipril compared with monotherapy with ramipril ${ }^{14}$. The composite primary endpoint of dialysis, doubling of the serum creatinine level, and death was reached at a higher rate in the combination therapy group. The rates of reaching the secondary endpoint of dialysis or doubling of the serum creatine level was also higher in the combination group. The incidence of chronic dialysis was similar across the 3 groups. Compared with monotherapy, combination therapy did decrease the rate of either the development of new microalbuminuria or the progression of microalbuminuria to macroalbuminuria ${ }^{14}$.

Most of these studies were of insufficient size and duration to properly assess the long-term safety issues surrounding the use of combination therapy. Further large-scale investigations are needed.

\section{Management of Hypertension}

The management of hypertension aims to prevent progressive CKD and control the development of CVD.

1) The target blood pressure is less than $130 / 80$ $\mathrm{mm} \mathrm{Hg}$, but when proteinuria is greater than $1 \mathrm{~g} /$ day, the target for blood pressure reduction becomes 125/75 mm Hg.

2) Because both nocturnal hypertension (nondipper) and morning hypertension can exacerbate CKD, home monitoring of blood pressure is a useful method for diagnosing them. Thus, home monitoring of blood pressure is important in antihypertensive therapy. Blood pressure should be gradually and carefully decreased over 2 to 3 months.

3) The first-line agents for controlling blood pressure are inhibitors of the RAS, such as ACEIs and ARBs. These drugs have been shown to have renoprotective effects (reducing proteinuria, 
decreasing glomerular hypertension, and slowing progression of nephrosclerosis) in addition to their ability to control blood pressure. When the blood pressure cannot be decreased, the combined use of a diuretic agent and a calcium channel blocker should be considered as a second-line treatment. In many cases, a multidrug regimen is required to reach targets of blood pressure reduction. Although the evidence does not support the direct effects of betablocking agents, alpha blocking agents, or central sympatholytic agents in preventing the progression of CKD, these drugs can also be expected to have beneficial effects in CKD by lowering blood pressure.

4) Improvements in lifestyle, especially a low-salt diet, are important, and a salt intake of less than 6 $\mathrm{g} /$ day is recommended. A low-salt diet enhances the antihypertensive effects of ACEIs and ARBs. When salt restriction is difficult, salt excretion can be increased with a low dose of a thiazide diuretic agent (CKD stage 1 to 3 ) or a loop diuretic (CKD stage 4 to 5 ). In elderly persons, appetite loss may result from excessive salt restriction, and care must be taken not to impair renal function through dehydration.

5) If a patient with CKD, especially that greater than stage 3 , is treated with an ACEI or ARB, serum creatinine levels might increase. Therefore, these agents should be administered at low dosages. Treatment is continued if the serum creatinine value increases by less than $30 \%$ of the most recent value or by less than $1 \mathrm{mg} / \mathrm{dL}$. When the increase is more than $30 \%$ of the most recent value or is greater than $1 \mathrm{mg} / \mathrm{dL}$, the dosage should be decreased or treatment should be stopped and a nephrologist should be consulted. Similar steps should be taken when the serum potassium level is greater than 5.5 $\mathrm{mEq} / \mathrm{L}$.

\section{References}

1. National Kidney Foundation: K/DOQI clinical practice guidelines for chronic kidney disease: evaluation, classification, and stratification. Am J
Kidney Dis 2002; 39: S1-S266.

2. Remuzzi G, Bertani T: Pathophysiology of progressive nephropathies. N Eng I Med J 1998; 339: 1448-1456.

3. Stevens LA, Greene T, Levey AS: Surrogate end points for clinical trails of kidney disease progression. Clin Am Soc Nephrol 2006; 1: 874-884.

4. Go AS, Chertow GM, Fan D, McCulloch CE, Hsu CY: Chronic kidney disease and the risk of death, cardiovascular events, and hospitalization. N Engl J Med 2004; 351: 1296-1305.

5. Nakayama M, Metoki H, Terawaki H: Kidney dysfunction as a risk factor for first symptomatic stroke events in a general Japanese population-the Ohasama study. Nephrol Dial transplant 2007; 22: 1910-1915.

6. K/DOQI Clinical practice guideline on hypertension and antihypertensive agents in chronic kidney disease. Am J kidney Dis 2004; 43: S1-S290.

7. Keith DS, Nichols GA, Gullion CM, Brown JB, Smith DH: Longitudinal follow-up and outcomes among a population with chronic kidney disease in a large managed care organization. Arch intern Med 2004; 164: 659-663.

8. Anavekar NS, McMurray JV, Velazquez EJ, et al: Relation between renal dysfunction and cardiovascular outcomes after myocardial infarction. N Engl J Med 2004; 351: 1285-1295.

9. Kim S, Izumi Y, Izumiya Y, et al.: Beneficial effects of combined blockade of ACE and AT I receptor on intimal hyperplasia in balloon-injured rat artery. Arterioscler Thromb Vasc Biol 2002; 22: 1299-1304.

10. Abe F, Omata K, Yamada M, et al.: Specific direct radioimmunoassay of angiotensin II (A II) in human plasma and the effects of angiotensin converting enzyme (ACE) inhibitor. Immunopharmacology 1999; 44: 199-204.

11. Urata H, Kinoshita A, Misono KS, et al.: Identification of a highly specific chymase as the major angiotensin II-forming enzyme in the human heart. J Biol Chem 1990; 265: 22348-22357.

12. Lewis EJ, Hunsicker LG, Clarke WR: Renoprotective effect of the angiotensin-receptor antagonist irbesartan in patients with nephropathy due to type 2 diabetes. N Eng J Med 2001; 345: 851-860.

13. Jennings DL, Kalus JS, Coleman CI, Manierski C, Yee J: Combination therapy with an ACE inhibitor and angiotensin receptor blocker for diabetic nephropathy: a meta-analysis. Diabet Med 2007; 24: 486-493.

14. Yusuf S, Teo KK, Pogue J, et al.: Telmisartan, ramipril, or both in patients at high risk vascular events. N Eng J Med 2008; 358: 1547-1559.

(Received, October 1, 2011)

(Accepted, November 30, 2011) 